\title{
Nuevos partidos en los Países Bajos y en España ¿Qué factores explican su surgimiento?
}

\author{
José Rama \\ Anne-Marie Reynaers \\ Universidad Autónoma de Madrid \\ jose.rama@uam.es; anne.reynaers@uam.es
}

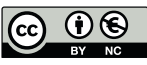

Recepción: 08-12-2017

Aceptación: 05-06-2018

Publicación: 19-02-2019

\section{Resumen}

El objetivo de este artículo es profundizar en las explicaciones sobre la emergencia de nuevos partidos. En concreto, se estudia qué cuestiones han sido centrales en los discursos de las nuevas formaciones y hasta qué punto dichos temas 1) son demandas de la sociedad (demand syde) y 2) ya habían sido abordados por los partidos establecidos (supply syde). Para ello, utilizando los casos de España y los Países Bajos, analizamos los programas electorales de las nuevas formaciones políticas, identificamos los temas principales de su discurso, con encuestas de opinión vemos las principales preocupaciones de españoles y holandeses y, gracias al Comparative Manifestos Project, comprobamos hasta qué punto estos temas no habían sido abordados previamente por las fuerzas tradicionales. Nuestros resultados muestran que, mientras que en los Países Bajos los temas de los nuevos partidos apenas son nombrados por las formaciones tradicionales, en España los asuntos centrales son compartidos por todas las formaciones, y los partidos emergentes surgen debido a los sentimientos de desconfianza hacia los que ya estaban establecidos.

Palabras clave: Comparative Manifestos Project; nuevos partidos políticos; programas electorales; España; Países Bajos

Abstract. New parties in the Netherlands and Spain: What factors explain their emergence?

The objective of this article is to shed light on explanations for the emergence of new parties. In particular, it examines which issues have been central to the discourses of these new formations and to what extent 1 ) these issues are relevant for the electors (demand side) and 2) have been addressed by already established parties (supply side). Using the cases of Spain and the Netherlands, we analyse the electoral programmes of the new parties, identify the main themes of their discourse and assess the main concerns for electors from Spain and the Netherlands based on opinion polls. Moreover, thanks to the Comparative Manifestos Project, we check to what extent these issues were not previously included in the manifestos of the established parties. Our results show that while the themes of new parties are scarcely named by traditional parties in the Netherlands, central issues are shared by all political formations in Spain, and new parties emerge due to feelings of distrust towards established ones.

Keywords: Comparative Manifestos Project; new political parties; electoral programmes; Spain; Netherlands 


\author{
Sumario \\ 1. Introducción \\ 4. Resultados \\ 2. Marco teórico \\ 5. Recapitulación y conclusiones \\ 3. Métodos y datos \\ Referencias bibliográficas
}

\title{
1. Introducción
}

A pesar de que la Gran Recesión ha afectado de manera muy distinta a las economías de los países de Europa occidental ${ }^{1}$, sus sistemas de partidos han cambiado de forma generalizada y con más intensidad desde entonces (Emanuele y Chiaramonte, 2016). Nuevas formaciones han entrado en los parlamentos nacionales al mismo tiempo que otras que ocupaban un segundo plano en la vida política han disfrutado de un mayor apoyo. Como consecuencia, la fragmentación de fuerzas y la volatilidad electoral han crecido (Dassonneville y Hooghe, 2017). Además, han cambiado las fórmulas de gobierno, al abrirse la estructura de competición de partidos (Mair, 1996, 2002), que en muchos de los países europeos estaba cerrada (Casal Bértoa y Weber, 2016).

Los estudios sobre nuevas formaciones políticas en democracias con sistemas de partidos consolidados se han centrado en determinar cuáles son las variables explicativas principales de su surgimiento (Harmel y Robertson, 1985; Tavits, 2006; Bolin, 2007; Hino, 2012). Así, y pese a que la mayoría de trabajos han abordado la importancia de factores de largo plazo, como el sistema electoral, la estructura de clivajes o la estabilidad del sistema de partidos, investigaciones recientes han señalado que los factores de corto plazo, como el contexto económico, es uno de los elementos principales que condicionan el surgimiento y apoyo a nuevas formaciones (Tavits, 2007; Arzheimer, 2009; Hernández y Kriesi, 2016). Aunque la relación entre la Gran Recesión y la emergencia de nuevos partidos podría parecer evidente, en los últimos años, sobre todo después de 2008, han surgido nuevos grupos políticos, tanto en escenarios de crecimiento como de recesión económica, sin mostrar una pauta general aparente.

En este sentido, dos casos de estudio, España y los Países Bajos, destacan por encima del resto. Por un lado, a causa de las muchas diferencias que presentan en su historia como democracias, en su diseño institucional y en su sistema de partidos ${ }^{2}$, además del modo como la reciente crisis afectó a sus

1. Sus efectos han sido mayores en los países del sur de Europa, Islandia e Irlanda. De hecho, la literatura especializada decidió dividir en «deudores» (Chipre, España, Eslovenia, Grecia, Hungría, Irlanda, Letonia, Portugal y Rumanía) y «acreedores» (Alemania, Austria, Bélgica, Dinamarca, Finlandia y Países Bajos) a los países de la Unión Europea.

2. El sistema de partidos de los Países Bajos podría considerarse multipartidista fragmentado. La fragmentación de partidos promedio ha sido, desde las primeras elecciones de 1952 hasta 2017, de 5,4 partidos. En los últimos comicios ha sido de 8,3 - uno de los más elevados de la serie histórica-. El nivel promedio de volatilidad electoral ha sido del 
economías ${ }^{3}$. Por otro lado, por las similitudes en el comportamiento político de sus electores y en los cambios que han sufrido sus sistemas de partidos en las recientes elecciones.

Ambos países experimentaron reacciones similares en las primeras elecciones que transcurrieron en el contexto de la Gran Recesión. En los Países Bajos, el partido Cristiano Demócrata (CDA) fue sustituido en el Gobierno por el partido de oposición, el Partido Popular por la Libertad (VVD), en las elecciones de 2010. En España, el Partido Popular (PP), en la oposición desde 2004, reemplazó en los comicios de 2011 al Partido Socialista Obrero Español (PSOE), hasta entonces en el Gobierno. En las posteriores elecciones, 2012 en los Países Bajos y 2015 en España, volvieron a ganar el VVD y el PP, respectivamente, pese a que en la mayoría de países europeos las malas condiciones económicas castigaban al partido del Gobierno y lo trasladaban a la oposición (Bermeo y Bartels, 2014; Magalhaes, 2014). En estos comicios, dos nuevas formaciones que manifestaban estar en contra del poder establecido (Torreblanca, 2015; Gómez Reino y Llamazares, 2015; Van Kessel, 2015; Schumacher y Rooduijn, 2013), el Partido de la Libertad (PVV) en los Países Bajos y Podemos en España, cosecharon un importante resultado electoral.

Además, los partidos socialdemócratas tradicionales de ambos países -el Partido Laborista (PvdA) y el PSOE - fueron los grandes perdedores de las elecciones, lo que subrayó la crisis de la socialdemocracia (Morgan, 2013; Roberts, 2017) ${ }^{4}$. Todo ello habría acontecido bajo unos niveles de volatilidad electoral sin precedentes y un aumento en los índices de fragmentación de partidos desconocidos hasta el momento, en buena medida justificados por la entrada de Ciudadanos (Cs) y Podemos en el Parlamento español, y de DENK y el Foro por la Democracia (FvD) en el de los Países Bajos.

13,4\%, siendo los comicios de 2017 unos de los más volátiles, con un 25\%. Los gobiernos de los Países Bajos han sido siempre de coalición. Por su parte, el sistema de partidos en España podría definirse como multipartidista con tendencia al bipartidismo. Sin embargo, las elecciones de 2015 dejaron una fragmentación electoral de 5,1 partidos, un valor muy por encima del promedio, que es del 3,7. Si analizamos los niveles de volatilidad electoral agregados entre elecciones, la cifra promedio desde 1977 hasta 2016 ha sido de 14,1; siendo los comicios de 2015 los segundos más volátiles, con un $35,5 \%$, solo superados por las elecciones de 1982.

3. Desde la Gran Recesión, la mayor caída del PIB en los Países Bajos llegó en el año 2009, con un $-3,8 \%$. Sin embargo, en la actualidad su crecimiento es estable, en torno al $2 \%$. Una evolución parecida experimentó España. De hecho, su mayor caída del PIB coincidió también en 2009 con un decrecimiento del -3,6\%, aunque para 2011, 2012 y 2013 proseguiría su descenso con valores del $-1 \%$, el $-2,9 \%$ y el $-1,7 \%$, respectivamente. Sin embargo, España creció en 2015 a un ritmo del 3,2\%. Donde no cabe duda de que la crisis afectó de forma distinta a los Países Bajos y España fue en los niveles de desempleo. Mientras que los comicios holandeses de 2010 se celebraron bajo un porcentaje de desempleados del $5 \%$, las elecciones españolas de 2011 lo hicieron con un $21,4 \%$ de personas sin ocupación, cifra que, en 2013, llegaría al 26,1\%. Sin embargo, en 2015 el paro había descendido en 4 puntos porcentuales.

4. En los últimos comicios celebrados en ambos países, 2016 en España y 2017 en los Países Bajos, el PvdA y el PSOE conocieron el peor resultado de su historia. 
La mayoría de investigaciones sobre nuevos partidos han optado por estudios de caso — predominantemente de carácter descriptivo- o por investigaciones de varios acontecimientos en las que, mediante el uso de técnicas cuantitativas, han explorado qué variables determinan su surgimiento. Sin embargo, hasta ahora se ha prestado poca atención al mensaje político de los partidos y, a nuestro juicio, esta es una de las principales razones por la que un elector decide dar su apoyo a una fuerza política u otra. En este estudio analizamos el mensaje de las nuevas formaciones para profundizar en la cuestión de por qué surgen en ambos países. Así, asumiendo que la Gran Recesión sirvió como ventana de oportunidad para la emergencia de nuevos partidos, podría ser posible que la mayor parte de temas incorporados por estos en sus discursos estuviera relacionada con aspectos económicos. Sin embargo, cabe esperarse también justo lo contrario: que otras cuestiones constituyan sus temas centrales. A nuestro juicio, si lo que queremos es averiguar por qué surgieron nuevos partidos en España y en los Países Bajos, debemos: 1) conocer las principales preocupaciones de los electores y 2) comparar los programas electorales de las nuevas formaciones con los programas de las que ya estaban establecidas. De esta forma estaríamos dando respuesta a la siguiente pregunta: ¿hasta qué punto los nuevos partidos abordan cuestiones en sus programas electorales que las fuerzas establecidas omitían, desatendiendo de este modo las demandas de los electores?

Este artículo se estructura en cuatro secciones. Primero, revisamos la literatura sobre nuevas formaciones y su surgimiento. Segundo, explicamos la metodología adoptada, basada en un análisis de contenido de los programas electorales de los nuevos partidos, lo que nos permite identificar los temas principales de cada uno de ellos. Tercero, a través del Comparative Manifestos Project (CMP), comparamos la presencia de estos temas en las formaciones tradicionales y en las nuevas 5 . Esta comparación por el lado de la oferta se completa con la identificación (en los estudios postelectorales) de los principales problemas de los electores (lado de la demanda). A continuación, describimos los resultados de los análisis. Por último, presentamos nuestras conclusiones.

\section{Marco teórico}

Esta sección se centra en dar respuesta a dos preguntas: 1) ¿qué es un nuevo partido? y 2) ¿por qué surgen nuevas formaciones?

Para establecer qué fuerzas políticas pueden considerarse nuevas ${ }^{6}$, utilizamos la definición propuesta por Mair (1999: 210), quien define un nuevo partido como aquel que, sin ser originalmente miembro del sistema que ya estaba consolidado, una vez accede a él es capaz de cambiar las dinámicas de

5. Para el caso de los Países Bajos, el CMP no ha incorporado la codificación de los programas electorales de las elecciones de 2017. Pese a esta ausencia, contamos con datos suficientes para dar respuesta a nuestras preguntas de investigación.

6. Para profundizar más en detalle sobre el concepto de partido nuevo, ver Sikk (2005: 397). 
competición establecidas hasta el momento. Además, tomamos como referencia a Harmel (1985) y Mair (1999), quienes distinguen tres tipos de nuevos partidos teniendo en cuenta la forma en la que surgieron:

1. Como consecuencia de la fusión de formaciones ya existentes.

2. Debido a una escisión.

3. Los partidos que no son producto de una fusión ni de una ruptura y que, por ello, pueden ser considerados genuinamente nuevos (Mair, 1999: 216).

El fenómeno de entrada de nuevas fuerzas políticas ha sido un tema de estudio recurrente en los trabajos sobre partidos y sistemas de partidos. En general, podemos agrupar en dos categorías las investigaciones sobre el surgimiento de nuevas formaciones en sistemas de partidos consolidados. Por un lado, estarían aquellas que han tomado a los factores de tipo institucional (los propias del sistema electoral) y a la estructura de clivajes del país como los principales elementos explicativos del surgimiento de nuevos partidos. Este tipo de factores son elementos del largo plazo. Por otro, se encontrarían las investigaciones que han considerado a las variables de tipo coyuntural (corto plazo) como las más adecuadas para explicar el éxito de las nuevas formaciones. Estos últimos trabajos se han centrado en el impacto del contexto económico o en los acontecimientos de carácter social y político.

Empezando por el primer tipo de investigaciones, Hauss y Rayside (1978) concluyen que los factores institucionales, las divisiones sociales y los partidos presentes en el sistema importan para el desarrollo de las nuevas formaciones, pero que, en todo caso, no constituirían un factor decisivo para la emergencia de nuevos grupos políticos. Bolin (2007), sin embargo, muestra que el sistema electoral es un factor clave para el éxito de las nuevas formaciones, dado que la relación entre el tamaño del Parlamento, la magnitud del distrito y la entrada de nuevos partidos es positiva y significativa. En esta misma línea, Hino $(2006,2012)$ advierte de la importancia del sistema electoral para el desarrollo exitoso de estas nuevas formaciones. En su estudio analiza el caso de los países europeos occidentales desde 1950 hasta 2004 centrándose en tres grupos de partidos (los de extrema derecha, los de la nueva política y los partidos étnico-regionalistas)

Dentro de los estudios centrados en factores de largo plazo, Harmel y Robertson (1985) consideran que los nuevos partidos surgen como respuesta a las nuevas necesidades políticas que demanda la sociedad. Las formaciones, por lo tanto, emergen en sistemas de partidos consolidados porque la aparición de nuevos temas, que podrían alterar las estructuras de clivajes establecidas, suscitaría el interés de los votantes. Trabajos más recientes han centrado sus esfuerzos en describir el modo en el que la importancia de nuevos asuntos (relacionados con la globalización, la inmigración y la Unión Europea) podría facilitar la entrada de partidos que diesen respuesta a estas nuevas cuestiones que las formaciones tradicionales no habrían sido capaces de incorporar a sus discursos (Kriesi et al., 2008; Hooghe y Marks, 2017). 
Por su parte, varias investigaciones sobre nuevos partidos se han centrado en factores de corto plazo. Pinard (1975) destaca el desempleo como la variable principal para explicar el surgimiento del Partido del Crédito en Quebec. En esta misma senda se sitúan los hallazgos de Tavits (2007: 118), para quien el paro, junto a otras variables de tipo institucional, explicaría el surgimiento de nuevas fuerzas en Europa del Este. Así lo señala:

[El] resultado más consistente de los estudios sobre emergencia de partidos en democracias avanzadas es el efecto de la economía en el corto plazo. Estudios previos han argumentado que la Recesión incrementa las entradas, porque provee a las élites de los nuevos partidos de una oportunidad para beneficiarse de los fallos de las políticas económicas adoptadas por los partidos establecidos. Cuando la economía no camina por la buena senda, los nuevos partidos tienen un tema claro y destacable sobre el que movilizar a los votantes, y los partidos existentes aparecen como menos creíbles.

Relacionado con lo anterior estaría el efecto de los sentimientos de desafección política que, además, son cada vez mayores entre los votantes europeos (Dalton y Weldon, 2006). Los electores, antes que abstenerse, habrían preferido dar su apoyo a nuevas formaciones, muchas de ellas con discursos en contra del poder establecido (Lago y Martínez, 2011: 7). De esta forma podría esperarse que, cuando las demandas de la sociedad no son canalizadas por las fuerzas ya existentes, un nuevo partido tenga la oportunidad de emerger. Este sería el caso de los partidos verdes, que entraron en los sistemas políticos establecidos para dar respuesta a cuestiones que las fuerzas tradicionales habrían descuidado (Mair, 1989).

Así, a priori, parecería que las explicaciones más apropiadas para justificar el surgimiento de nuevas formaciones en las recientes elecciones de España y de los Países Bajos, con los casos de Podemos y Ciudadanos, FvD, DENK, PVV, 50Plus y PvdD, podrían estar relacionadas tanto con factores de largo como de corto plazo. Por un lado, cabe suponer que la proporcionalidad del sistema electoral holandés sea un escenario favorable para que nuevas formaciones vean la posibilidad de conseguir un éxito electoral relativo (Beyens et al., 2015: 3). Con una barrera legal y real de entrada del 0,67 \% y una magnitud de distrito de 150 escaños, las posibilidades de éxito son mayores que en España, con una barrera legal (no real) de entrada del 3\% a nivel de circunscripción y con una magnitud de distrito media de 6,7 escaños. Además, ambos países presentan sistemas de partidos que arrojan valores de más de dos dígitos en su volatilidad electoral — como señalábamos anteriormente, el promedio de los Países Bajos es del 13,4\%, mientras que el de España es del 14,1\%- (Linz y Montero, 2000; Rama, 2016; Mair, 2008). Bajo estas circunstancias un nuevo partido podría tener ciertas facilidades para entrar en la competición electoral. Por otro lado, parece que nuevas fracturas sociales hayan cambiado el comportamiento de los votantes. En España, al igual que en Italia y en Grecia, los jóvenes con mayor nivel educativo presentan un comportamiento electoral diferenciable (Segatti y Capuzzi, 2016). En los Países Bajos los temas relacio- 
nados con la inmigración (Boomgaarden y Vliegenthart, 2007; Vliegenthart et al., 2012) y la Unión Europea (De Vries, 2007) han podido romper los esquemas tradicionales de voto.

Si nos centramos en los factores de corto plazo, como la situación política y económica, estos podrían haber actuado como ventana de oportunidad para desencadenar las transformaciones que latían desde hacía tiempo en los sistemas de partidos de ambos países. Por un lado, la Gran Recesión ha propiciado que los electores se sientan atraídos por los partidos extremos y no tradicionales, muchos de ellos, nuevos (Lindvall, 2014; Hernández y Kriesi, 2016). Y, por otro lado, la desafección con las formaciones establecidas y con la política en general podría estar detrás del surgimiento de nuevos grupos, sobre todo en el caso español (Orriols y Cordero, 2016; Bosch y Durán, 2017).

Nuestro estudio comparado de los nuevos partidos de un país del sur de Europa, España, y de otro de Europa occidental, los Países Bajos, desde una nueva perspectiva metodológica (el análisis de contenido de los programas electorales), busca señalar cuáles fueron los principales temas incorporados en sus programas. Además, una vez identificados, lo que pretendemos es advertir hasta qué punto estos temas se encuentran, o no, incorporados en los programas electorales de los partidos establecidos y hasta qué punto suscitan el interés de los votantes, quienes, al ver que sus demandas no son satisfechas por las fuerzas tradicionales, pueden sentirse atraídos por las nuevas formaciones (Mair, 1989).

Nuestras premisas apuntan a que los nuevos partidos, tanto en España como en los Países Bajos, han aprovechado el contexto de crisis económica para emerger en sistemas de partidos consolidadas, debido tanto a la crisis del sistema partitocrático (Mair, 2015), como al surgimiento de nuevos temas en los que los nuevos partidos se posicionan mejor que las formaciones tradicionales (Hooghe y Marks, 2017). En este sentido, esperamos que las nuevas fuerzas incorporen en sus programas electorales mayoritariamente cuestiones relacionadas con conflictos de interés surgidos últimamente o relativas a la mejora de la calidad democrática, que, a su vez, no son cuestiones centrales de los discursos de los partidos tradicionales, pero que, sin embargo, tienen importancia para los votantes.

\section{Métodos y datos}

Siguiendo a Klingemann et al. (1994), consideramos en nuestro análisis los programas electorales de las nuevas formaciones, es decir, aquellas que han surgido en recientes elecciones en España y en los Países Bajos y que en la actualidad tienen representación en los parlamentos nacionales. Los programas electorales recogen «una mezcla de declaraciones ideológicas, principios abstractos, metas generales y dimensiones políticas específicas [...] contienen un énfasis relativo en ciertas ideas, políticas, cuestiones y preocupaciones seleccionadas por los líderes del partido» (Klingemann et al., 1994: 23-27). 
Tabla 1. Características de los nuevos partidos políticos en España (SP) y los Países Bajos (PB)

\begin{tabular}{llllllll}
\hline Partido & 50plus & Cs & DENK & FVD & Podemos & PvdD & PVV \\
País & PB & SP & PB & PB & SP & PB & PB \\
Año fundación & 2009 & 2006 & 2015 & 2015 & 2014 & 2002 & 2004 \\
Escaños 2017 & 4 & 32 & 3 & 3 & 67 & 5 & 20 \\
Orientación & Liberal & Liberal & Liberal & Conservador & Comunista & Respeto a & Conservador \\
& & & & y populista & los animales \\
Emergencia & Nuevo partido & Nuevo partido & Escisión & Nuevo partido & Nuevopartido & Nuevo partido Escisión \\
\hline
\end{tabular}

Fuente: elaboración propia en base a http://www.parlgov.org

El análisis de contenido tiene dos objetivos principales. Primero, identificar y comparar el mensaje central de los nuevos partidos políticos y, segundo, comparar los temas abordados por estos con los utilizados por las formaciones tradicionales. Siguiendo los criterios establecidos por Mair (1999) y Hamels (1985), consideramos a Podemos y a Ciudadanos como nuevas formaciones españolas ${ }^{7}$, y al Partij voor de Vrijheid (PVV), Foro para la Democracia (FvD), DENK (Piensa en holandés, Igualdad en turco), Partij voor de Dieren (PvdD) y 50plus como nuevas formaciones holandesas ${ }^{8}$. En la tabla 1 se resumen las características de los nuevos partidos políticos.

Con el fin de captar la esencia central del mensaje de los nuevos partidos políticos y las diferencias existentes entre ellos, exploraremos sus programas electorales mediante el análisis cualitativo (Krippendorff, 2004) ${ }^{9}$.

Las unidades de análisis son sus programas electorales. Incluimos los que fueron previos a las elecciones que se celebraron bajo la crisis económica y en las cuales aquellas formaciones obtuvieron representación parlamentaria. La tabla 2 ofrece una visión general de los programas electorales seleccionados por partido político. Optamos por analizarlos en lugar de estudiar los discursos de sus líderes u otro tipo de manifestación pública porque asumimos que ofrecen

7. No incluimos a la formación Unión Progreso y Democracia (UPyD). Pese a ser un nuevo partido que surgió en España en 2007, obteniendo representación en las elecciones generales de 2008 y 2011, en los comicios de 2015 y 2016 no consiguió ningún escaño en el Congreso de los Diputados. Asimismo, y pese a que consideramos a Ciudadanos como un nuevo partido, dado que las elecciones generales de 2015 fueron las primeras en las que compitió realmente por entrar en el Parlamento, vale la pena señalar que su origen en 2006 está circunscrito a la Comunidad Autónoma de Cataluña, donde obtuvo por primera vez representación en los comicios autonómicos de este mismo año.

8. Excluimos del análisis a la Unión Cristiana (CU). Pese a que no pertenece al sistema de partidos original, es una formación que habría obtenido representación ya en las elecciones de 2002. Además, es el resultado de la unión entre la Liga Política Reformada (GPV) — fundada en 1948 - y la Federación Política Reformatoria (RPF) —fundada en 1975.

9. La palabra cualitativo hace referencia a la naturaleza no numérica de los datos. Contrariamente al análisis de contenido cuantitativo, que se «utiliza para comparar la aparición de contenido totalmente manifiesto", el análisis cualitativo se detiene en el significado de datos no numéricos y es, por lo tanto, más útil para los fines de nuestro estudio (Rourke y Anderson, 2004). 
Tabla 2. Características de los programas seleccionados por partido político

\begin{tabular}{lll}
\hline Partido & Año & Número de páginas \\
\hline 50plus & 2012,2017 & 12,17 \\
Cs & 2015 & 338 \\
DENK & 2017 & 76 \\
FvD & 2017 & Página web \\
Podemos & 2015 & 332 \\
PvdD & $2006,2010,2012,2017$ & $45,56,42,38$ \\
PVV & $2006,2010,2012,2017$ & $6,60,56,1$ \\
\hline
\end{tabular}

Fuente: elaboración propia, acceso a los programas electorales en: https://bit.ly/2L5RU6c

mejor y de manera más compacta la esencia del discurso. Todos los programas electorales están disponibles en Internet, a excepción del presentado por el FvD, que puede consultarse por temas a través de su página web.

$\mathrm{El}$ análisis de datos cualitativos es un proceso mediante el cual se organizan los datos en categorías para identificar patrones y relaciones entre estas categorías (McMillan y Schumacher, 1993) asignando códigos a fragmentos de texto (Strauss y Corbin, 1998). Los códigos se derivan de los datos mismos o de la teoría, dependiendo de si el análisis adopta un enfoque inductivo o deductivo (LeCompte y Schensul, 1999). En este examen hemos utilizado códigos preestablecidos para analizar los programas en función de las siguientes dimensiones:

1. Qué temas abordan y qué dicen acerca de ellos.

2. El orden en que se tratan los diferentes temas (cabe pensar que los primeros que se discuten son los que consideran más importantes).

3. Las referencias explícitas a temas o a valores presentados como importantes por los propios partidos.

4. La forma en que, al discutir estos temas, se hace referencia explícita a los puntos de vista de otras formaciones.

5. Qué valores se utilizan para definir el discurso de los partidos.

Así, una vez identificados los temas principales que los nuevos partidos abordan en sus programas políticos, buscamos testar hasta qué punto las formaciones establecidas no recogen dichos temas en sus manifiestos electorales. Para ello nos valemos del Comparative Manifestos Project (CMP). Se trata de un proyecto cooperativo de largo plazo basado en la codificación de los programas electorales de los partidos como forma de medir la relevancia de las diferentes cuestiones dentro del espacio político, así como la posición de las distintas formaciones sobre estos temas. Contiene información de más de 50 países que cubren todas las elecciones libres y democráticas desde $1945^{10}$.

10. Tres son las ventajas de usar los programas electorales de las diversas formaciones para medir su mensaje político: 1) son publicados por los órganos oficiales del partido y representan la visión de su conjunto, no de un individuo; 2) son publicados en cada elección, eso nos permite ver su evolución en el tiempo, y 3) con ellos podemos comparar el tratamiento de los temas con otros partidos (Alonso et al., 2012). Ver <www.manifesto-project.wzb.eu>. 
Esta comparación nos permitirá advertir hasta qué punto, tal y como sugiere la literatura, los nuevos partidos surgen porque la oferta de formaciones existente no cubre las demandas de los ciudadanos. Asimismo, y para dar respuesta a cuáles son las demandas de los ciudadanos, nos valdremos de distintas encuestas de opinión. En España emplearemos el estudio postelectoral de 2015 del Centro de Investigaciones Sociológicas (CIS), y en los Países Bajos, las encuestas postelectorales del Dutch Parliamentary Election Studies, en las que se pregunta por los principales problemas de los ciudadanos.

Con todo ello estaríamos dando respuesta a dos cuestiones. Por un lado, identificaríamos qué temas, de corto o largo plazo, son los que mayoritariamente incorporan en sus programas políticos las nuevas formaciones y, por otro, comprobaríamos hasta qué punto estos temas, que son principales en el discurso de los nuevos partidos, 1) no han sido tratados por los partidos establecidos y 2) suscitan interés entre el electorado.

\section{Resultados}

\subsection{Temas de los nuevos partidos en los Países Bajos}

La tabla 3 resume los cinco principales temas que las nuevas formaciones de los Países Bajos incorporaron en sus programas electorales en cada una de las elecciones analizadas. Las cuestiones compartidas por los diferentes grupos políticos son la regeneración democrática, el islam, la inmigración, la Unión Europea y la soberanía nacional. La regeneración democrática (una democracia más directa) es una preocupación de casi todas las nuevas formaciones. FvD, por ejemplo, propone el uso de referéndums vinculantes, la elección directa de alcaldes y del primer ministro y la participación política (incluido el voto) a través de Internet.

La inmigración (y sus consecuencias negativas para la sociedad, según apuntan los partidos) es un tema de interés para el FvD, 50Plus y el PVV. Así, el $\mathrm{FdV}$ propone el control fronterizo y la prohibición de la entrada de inmigrantes económicos. Las propuestas del PVV van en una línea similar: rechazo a todos los solicitantes de asilo y cierre de las fronteras. Los tres partidos también prestan especial atención al islam y al impacto que ejerce en la cultura holandesa. 50plus, por ejemplo, está explícitamente en contra de la introducción de la sharía (la ley islámica) y afirma que la fe en el islam debería desarrollarse únicamente en el espacio privado (vida personal), sin repercutir en el ámbito público. Además, propone la protección del modo de vida, de la cultura y del idioma holandés. $\mathrm{FvD}$, de igual manera, sostiene que hay que resguardar la cultura europea y proteger, por ley, los valores holandeses. El PVV manifiesta directamente su deseo de poner fin a la «islamización» de la sociedad holandesa y a la inmigración desde los países musulmanes. Para ello propone el cierre de mezquitas y de escuelas islámicas, así como la prohibición del Corán. Además, demandan la introducción de un nuevo primer artículo de la Constitución para establecer que las culturas cristiana, judía y humanista sean dominantes. 
Tabla 3. Cinco temas principales de los nuevos partidos en los Países Bajos, 2006-2017

\begin{tabular}{|c|c|c|}
\hline Partido & Elección & Temas \\
\hline \multirow[t]{2}{*}{ 50plus } & 2012 & $\begin{array}{l}\text { Islam, Unión Europea, posición socioeconómica de los mayores, } \\
\text { sistema sanitario, tamaño del Estado. }\end{array}$ \\
\hline & 2017 & $\begin{array}{l}\text { Inmigración, posición socioeconómica de los mayores, protección } \\
\text { de la cultura y el idioma holandeses, regeneración democrática } \\
\text { (democracia directa), sistema sanitario. }\end{array}$ \\
\hline DENK & 2017 & Discriminación, diversidad, igualdad, racismo, solidaridad. \\
\hline FvD & 2017 & $\begin{array}{l}\text { Regeneración democrática (democracia directa), inmigración, } \\
\text { nacionalismo de la UE, renovación del sistema político, soberanía } \\
\text { nacional. }\end{array}$ \\
\hline \multirow[t]{4}{*}{ PvdD } & 2006 & $\begin{array}{l}\text { Bienestar animal, desarrollo personal, ecosistema, respeto mutuo, } \\
\text { sostenibilidad. }\end{array}$ \\
\hline & 2010 & $\begin{array}{l}\text { Bienestar animal, compasión, ecosistema, protección de minorías, } \\
\text { sostenibilidad. }\end{array}$ \\
\hline & 2012 & $\begin{array}{l}\text { Bienestar animal, confianza en el Gobierno, cooperación internacio- } \\
\text { nal, crisis económica, ecosistema. }\end{array}$ \\
\hline & 2017 & Bienestar animal, economía, ecosistema, empleo, sostenibilidad. \\
\hline \multirow[t]{4}{*}{ PVV } & 2006 & $\begin{array}{l}\text { Inmigración, islam, multiculturalismo, regeneración democrática } \\
\text { (democracia directa), soberanía nacional. }\end{array}$ \\
\hline & 2010 & $\begin{array}{l}\text { Inmigración, islam, multiculturalismo, protección de los homosexuales } \\
\text { y de las mujeres, seguridad. }\end{array}$ \\
\hline & 2012 & $\begin{array}{l}\text { Estado de bienestar, inmigración, islam, nacionalismo de la UE, } \\
\text { soberanía nacional. }\end{array}$ \\
\hline & 2017 & $\begin{array}{l}\text { Inmigración, islam, nacionalismo de la UE, regeneración democráti- } \\
\text { ca (democracia directa), terrorismo. }\end{array}$ \\
\hline
\end{tabular}

Fuente: elaboración propia en base a los programas electorales de los partidos.

Mientras que para el PVV la inmigración y el islam son temas que resultan claves, para 50Plus y el FvD se trata de cuestiones de segundo orden. El primero de ellos se centra principalmente en la posición socioeconómica de los mayores. 50Plus demanda el restablecimiento de la edad de jubilación a los 65 años, la supresión de recortes de las pensiones, el estímulo a los trabajadores mayores a seguir activos laboralmente, la ayuda a los ancianos desempleados a encontrar empleo y el aumento del poder adquisitivo de los trabajadores de mayor edad. El FvD, por su parte, centra su discurso sobre todo en la regeneración democrática y en la renovación del sistema político. La soberanía nacional y su relación con el nacionalismo de la UE son temas compartidos por el PVV y el FvD. Este último quiere realizar un referéndum sobre la pertenencia a la UE, mientras que el PVV propone directamente la salida de Holanda. 50Plus, por el contrario, apoya seguir formando parte de la UE, aunque sí considera necesario reducir la participación económica. 
Los partidos cuyos temas no coinciden con los anteriores son DENK y PvdD. Los líderes de DENK, Tunahan Kuzu y Selçuk Öztürk son holandeses de origen turco que hasta 2014 formaron parte del Partido Laborista (PvdA). Frente al rechazo de las culturas, religiones o valores distintos a los holandeses (expresado por el PVV, el FvD y 50Plus), DENK propone la aceptación de esa diversidad y lucha contra la discriminación y el racismo. Dicha formación quiere una sociedad inclusiva, la igualdad de trato de los ciudadanos con independencia de su origen y la aceptación y el respeto hacia la diversidad. A través de medios democráticos, DENK quiere contrarrestar a los "políticos oportunistas que crean miedo y odio" en la sociedad (p. 8) y ofrecer igualdad de oportunidades formativas, además de asegurar la aceptación y la atención a la diversidad en el sistema educativo.

Los temas propuestos por el PvdD son totalmente diferentes a los planteados por el resto de nuevas formaciones. Los principales asuntos que aborda son el bienestar animal y el ecosistema. El PvdD defiende los derechos de los animales, la mejora de su bienestar y el rechazo a su abuso. En cuanto al ecosistema, propone medidas para su protección y sostenibilidad. En los programas electorales analizados se presta especial atención a estos dos temas, mientras que el espacio para asuntos generales es mucho menor. No obstante, el distinto peso de esos dos bloques se va igualando con el tiempo. En el programa electoral de 2010, por ejemplo, las 40 primeras páginas trataban cuestiones relacionadas con el ecosistema y el bienestar animal, dejando las últimas diez para abordar temas como la educación, la seguridad y la cultura (una línea similar a la que siguen en 2012, aunque en este programa dedican un mayor espacio a la cooperación internacional, la confianza en el Gobierno y la crisis económica). Finalmente, el programa electoral de 2017 experimenta un giro con respecto a los textos precedentes: comienza abordando temas económicos y relacionados con el empleo, y no habla tanto del bienestar animal ni del ecosistema.

\subsection{Temas de los partidos tradicionales y preocupaciones ciudadanas en los Paises Bajos}

La tabla 4, que cubre las elecciones que tuvieron lugar en los Países Bajos desde 2006 hasta 2012 y que contiene información sobre los partidos que consiguieron representación en dichos comicios ${ }^{11}$, muestra el porcentaje de alusiones que contienen los programas electorales de las formaciones en referencia a cada uno de los temas identificados. De nuestro análisis anterior hemos extraído cinco aspectos principales por cada partido. Con ello podemos afirmar que son ocho las mayores cuestiones que destacan las nuevas formaciones: el bienestar social; la mejora democrática; la libertad y los derechos humanos; el medioambiente y el respeto a los animales; la protección de la cultura y de los valores nacionales; los problemas de soberanía nacional; la inmigración, y la justicia, la ley y el orden. Para medir estos ocho temas nos valemos de los ocho puntos del CMP que recoge la tabla 4. El CMP, como hemos adelantado en la introducción, no 
Tabla 4. Porcentaje de alusiones a distintos temas por partido en los Países Bajos, 2006, 2010 y 2012 1, 2

\begin{tabular}{|c|c|c|c|c|c|c|c|c|}
\hline & $\begin{array}{l}\text { Estado del } \\
\text { bienestar }\end{array}$ & $\begin{array}{c}\text { UE } \\
\text { negativa }\end{array}$ & Democracia & $\begin{array}{l}\text { Libertad y } \\
\text { derechos } \\
\text { humanos }\end{array}$ & $\begin{array}{l}\text { Protección del } \\
\text { medio ambiente }\end{array}$ & $\begin{array}{c}\text { Modo de vida } \\
\text { nacional: positivo }\end{array}$ & $\begin{array}{l}\text { Ley y } \\
\text { justicia }\end{array}$ & $\begin{array}{l}\text { Multiculturalismo: } \\
\text { negativo }\end{array}$ \\
\hline $\mathrm{GL}$ & $14,419,111,6$ & 0,300 & $2,72,33,9$ & $4,512,68,5$ & $9,37,310,3$ & $0,60,40,2$ & $3,52,33$ & $1,41,30,4$ \\
\hline SP & $13,617,315,2$ & $2,10,81,6$ & $4,15,53,7$ & $1,63,62,1$ & $5,14,27,9$ & $0,70,40,1$ & 566,7 & $0,70,40,2$ \\
\hline PvdA & $15,116,313,3$ & 100,2 & $2,232,5$ & $2,73,43$ & $2,722,9$ & $0,80,11,4$ & $7,64,95$ & $1,41,31$ \\
\hline D66 & $9,18,88$ & $0,300,1$ & $4,432,4$ & $7,47,67,7$ & $5,64,43,8$ & $0,200,2$ & $3,442,9$ & $0,50,61$ \\
\hline WD & $7,46,65,2$ & $0,21,31,9$ & $30,80,7$ & $3,75,64,5$ & $2,820,9$ & $2,90,40,5$ & $12,7910,6$ & $1,33,92,3$ \\
\hline CDA & 119,410 & $0,80,21,1$ & $2,31,11,3$ & $1,22,34,6$ & $2,23,71,6$ & $1,90,91,3$ & $5,77,35,4$ & $1,11,61$ \\
\hline CU & $7,97,16$ & $1,70,62,8$ & $10,70,9$ & $2,23,74,2$ & $7,95,85,6$ & $0,50,80,4$ & $4,15,85,1$ & $0,91,61,5$ \\
\hline PVV & $2,97,85$ & $5,85,319,6$ & $3,72,82$ & $2,91,31,9$ & $2,94,12$ & $84,14,3$ & $19,715,510,7$ & $15,313,611,2$ \\
\hline PvdD & 346 & $00,10,8$ & 113,3 & $1,52,44,3$ & 626241,5 & 000,1 & $5,74,22,7$ & $1,50,10,5$ \\
\hline SGP & $10,9124,7$ & $0,71,52,1$ & $0,30,60,3$ & $1,92,81$ & $2,62,42,6$ & $1,40,30,4$ & $6,68,97,3$ & $2,722,6$ \\
\hline
\end{tabular}

1. En negro, los datos correspondientes al año 2006; en rojo, los de 2010, y en verde, los de 2012.

2. Los indicadores utilizados son los de bienestar per110, per202, per201, per501, per601, per605 y per608.

Fuente: elaboración propia en base a los datos del CMP.

tiene información sobre las últimas elecciones celebradas en 2017, con lo que no podemos testar hasta qué punto los temas que destacan los nuevos partidos fueron incorporados por las formaciones tradicionales en estas elecciones.

Por lo general, los nuevos partidos, el PVV y el PvdD, destacan en sus discursos temas que tienen una menor relevancia para las formaciones tradicionales o que, en alguno de los casos, están totalmente excluidos en sus programas políticos. Así ocurre con la defensa de la cultura nacional, la defensa de la soberanía ante la absorción de competencias de la UE, el medio ambiente y el respeto a los animales y la inmigración.

La mayor inclusión de estas cuestiones en los programas de los nuevos partidos vendría justificada por las demandas de los ciudadanos. Si tomamos los estudios postelectorales del Dutch Parliamentary Election Studies ${ }^{12}$ vemos como en 2006 los temas que más preocupaban a los ciudadanos eran, por este orden, la seguridad social, las minorías y la inmigración, el crimen y la seguridad, la educación y la pobreza. En 2010 eran la economía, las minorías, el crimen, la política y las normas y valores, mientras que en 2012 eran la economía, la seguridad social, el empleo, los ingresos y los impuestos y las minorías. Además, pese a no estar entre las cinco primeras preocupaciones de los holandeses, en la lista de problemas el medio ambiente ocupaba las posiciones 13 en 2010 y 2012 y 16 en 2006. Por lo tanto, del análisis se podría desprender que los nuevos partidos de los Países Bajos habrían surgido como respuesta a las nuevas cuestiones que suscitan interés entre parte del electorado, los cuales habrían tenido un tratamiento menor en los discursos de las formaciones tradicionales.

12. Enlace en línea: <http://www.nkodata.nl>. Se ha utilizado la pregunta sobre el «principal problema para el país» v041 para 2006 y 2012, y v071 para 2012. 


\subsection{Temas de los nuevos partidos en España}

En el caso de España, la tabla 5 resume los principales temas que contienen los programas electorales de los nuevos partidos. Los asuntos centrales abordados por Cs son la eficiencia del sector público; la transparencia política, administrativa y jurídica; la selección y la promoción basadas en el mérito; la regeneración democrática, y la cooperación y la coordinación multinivel. Para mejorar la eficiencia se propone, entre otras medidas, controlar el gasto público y reducir tanto el tamaño como el alcance de la burocracia y la Administración. Cs incide en la importancia de generar servicios públicos más eficientes, eficaces, innovadores y competitivos. Por otra parte, la importancia que Cs otorga a los temas relacionados con la transparencia, o la selección y la promoción basadas en el mérito, estaría justificada por la necesidad de hacer frente a la corrupción política y administrativa. Así, establecer sistemas meritocráticos aumentaría la imparcialidad de las instituciones públicas, lo que, en última instancia, supondría un paso hacia la despolitización. En cuanto a la regeneración democrática, un tema también abordado por Podemos, Cs presenta varias propuestas para mejorar la democracia, la representatividad y la participación. En lo relativo a la cooperación y a la coordinación multinivel, Cs apuesta por una mayor y una mejor coordinación entre los diferentes niveles de gobierno, así como por la creación de pactos nacionales con respecto, entre otros asuntos, al sistema educativo y al desarrollo científico. Este partido, además, apoya la adhesión de España a la Unión Europea y persigue la cooperación y la unidad entre las diferentes regiones españolas. Podemos, en cambio, reconoce el carácter plurinacional de España y el derecho a decidir democráticamente el tipo de relación que se desee establecer con el conjunto del país.

Los temas sobre los que pone el foco Podemos son el bienestar social, la eficiencia energética, la modernización de la economía, la solidaridad y la regeneración democrática. Para dicha formación política, este último tema supone la implementación de medidas que ayuden a fomentar una democracia más directa. Para Podemos esta es una de las principales acciones necesarias para resolver muchos de los conflictos que afectan a la sociedad, a la política y a la economía españolas, como son la crisis económica, el paro, la corrupción y la desigualdad política. Podemos propone el uso de referéndums y la participación ciudadana para formular, implementar y evaluar políticas públicas; la igualdad política a través de la reforma del sistema electoral, y un incremento

Tabla 5. Cinco temas principales de los nuevos partidos en España, 2015

\begin{tabular}{lll}
\hline Partido & Elección & Temas \\
\hline Ciudadanos & 2015 & $\begin{array}{l}\text { Cooperación y coordinación multinivel, eficiencia, meritocracia, } \\
\text { regeneración democrática (democracia directa), transparencia. }\end{array}$ \\
Podemos & 2015 & $\begin{array}{l}\text { Bienestar social, eficiencia energética, modernización económica, } \\
\text { regeneración democrática (democracia directa), solidaridad. }\end{array}$
\end{tabular}

Fuente: elaboración propia en base a los programas electorales de los partidos. 
Tabla 6. Porcentaje de referencias a cada uno de los temas en los programas electorales, elecciones generales $2015^{1}$

\begin{tabular}{|c|c|c|c|c|c|c|c|}
\hline & $\begin{array}{l}\text { Estado del } \\
\text { bienestar }\end{array}$ & Democracia & Corrupción & $\begin{array}{l}\text { Eficiencia } \\
\text { del Gobierno y de } \\
\text { la Administración }\end{array}$ & $\begin{array}{l}\text { Libertad y } \\
\text { derechos } \\
\text { humanos }\end{array}$ & $\begin{array}{l}\text { Protección } \\
\text { del medio } \\
\text { ambiente }\end{array}$ & $\begin{array}{c}\text { Tecnología e } \\
\text { infraestructuras }\end{array}$ \\
\hline UP & 29,8 & 5,4 & 0,7 & 1,3 & 3,1 & 9,3 & 4,8 \\
\hline Podemos & 17,8 & 7,2 & 2,5 & 8,5 & 4 & 6,7 & 8,5 \\
\hline PSOE & 21,7 & 4,9 & 2,8 & 5,4 & 3,4 & 1,4 & 9,5 \\
\hline Cs & 12,6 & 1,6 & 2,4 & 12,3 & 2 & 4,7 & 12,5 \\
\hline PP & 16 & 3,7 & 1,7 & 6 & 2,9 & 1,8 & 11,2 \\
\hline
\end{tabular}

1. Los indicadores utilizados son los de bienestar, per201, per202, per303, per304, per411 y per501.

Fuente: elaboración propia en base a los datos del CMP.

de la participación de los trabajadores en la gestión de las empresas. En relación con el bienestar social, este partido quiere garantizar constitucionalmente derechos sociales y proponer medidas que ayuden a reducir los niveles de desigualdad. Defienden la introducción de un plan de bienestar social, una renta garantizada, una reversión de los recortes sufridos desde la crisis económica, la garantía y la protección de la salud, la educación, la vivienda, el trabajo digno y el transporte público. Podemos quiere fomentar la solidaridad mediante acciones como el establecimiento de impuestos de solidaridad a las entidades financieras privadas y la creación de un eurogrupo social. En relación con la modernización de la economía, quiere luchar contra el desempleo y la precariedad laboral (introduciendo el derecho al trabajo), además de fomentar una economía social, una economía del bien común y una economía colaborativa.

\subsection{Temas de los partidos tradicionales y preocupaciones ciudadanas en España}

La tabla 6 contiene el porcentaje de alusiones que recogen en sus programas electorales las formaciones de ámbito nacional sobre los temas que hemos identificado como los principales de los nuevos partidos. Al contrario de lo que veíamos en el caso de los Países Bajos, no parece que estas cuestiones sean «monopolio» de las nuevas formaciones. Pese a todo, sí podríamos destacar que, por ejemplo, Podemos es el partido que más alusiones hace a los temas relacionados con la democracia y uno de los que más habla sobre la eficiencia administrativa y la mejora en la calidad de gobierno. Justo en este punto es en el que más destaca Ciudadanos, al igual que en la mejora tecnológica y de las infraestructuras.

Por el lado de la demanda, la encuesta postelectoral del CIS de $2015^{13}$ destaca que los problemas que más preocupan a los españoles son, por este orden,

13. Estudio CIS 3126. Se puede consultar en <http://www.cis.es/cis/opencm/ES/1_encuestas/ estudios/ver.jsp?estudio $=14258>$. Hemos utilizado la pregunta P301 sobre los principales problemas de los españoles. 
el paro, la corrupción, la economía, el terrorismo internacional, la sanidad y la educación. Estos temas son abordados tanto por los nuevos partidos como por las formaciones tradicionales. Así, parecería más bien, en contra de lo que hemos visto en el caso de los Países Bajos, que los nuevos partidos en España no habrían surgido tanto a causa de que la oferta existente no cubría las demandas de los ciudadanos, sino más bien debido a que la oferta existente no seducía ya a los ciudadanos. Esto estaría apuntando a un proceso de desenganche del electorado con las viejas fuerzas políticas (Mair, 2015), pero no tanto al surgimiento de nuevos temas que susciten interés entre los votantes, no incorporados por las formaciones tradicionales y sí tratados en los programas de los nuevos partidos.

\section{Recapitulación y conclusiones}

Tras el estudio de los programas electorales de los nuevos partidos en España y en los Países Bajos, así como la identificación de los temas principales que estos abordan, hemos podido comprobar hasta qué punto estos temas: 1) no habían sido incorporados en el programa electoral de los partidos establecidos (gracias al CMP), y 2) suscitaban el interés de los electores (gracias a las encuestas postelectorales).

Los temas centrales de los nuevos partidos en los Países Bajos podrían resumirse en torno a estas diez cuestiones: la inmigración, la discriminación, la (des)islamización, el multiculturalismo, la Unión Europea, el bienestar animal, el ecosistema, la soberanía nacional, la regeneración democrática y la protección de la situación socioeconómica de las personas mayores de 50 años. Frente a ello, en España las cuestiones clave sobre las que giran los programas de los nuevos partidos son el desempleo, el modelo económico, la corrupción y el fraude, la despolitización de las instituciones públicas, la cooperación y la coordinación entre los diferentes niveles de gobierno, el medioambiente, la participación y la colaboración ciudadana, la igualdad y la solidaridad, la regeneración democrática, el bienestar social y la transparencia política, administrativa y jurídica.

En el caso de los Países Bajos, las dimensiones principales de los nuevos partidos apenas son incorporadas por las formaciones tradicionales en sus programas electorales. En España, en cambio, no parece existir una diferencia significativa entre nuevos y viejos partidos en el porcentaje de alusiones a los temas que hemos identificado como principales de las nuevas fuerzas políticas. Los temas mencionados por los partidos españoles parecen ser de carácter estructural, como, por ejemplo: la regeneración democrática, el funcionamiento de las instituciones públicas, la economía y las dinámicas entre los diferentes niveles de gobierno. Así, los nuevos partidos habrían surgido como respuesta a los fallos del sistema político establecido, para mejorar los canales de participación democrática, poner freno a los casos de corrupción política, hacer más eficientes a las administraciones públicas y, en general, despolitizar la política (Orriols y Cordero, 2016; Lavezzolo y Ramiro, 2017; Vidal, 2017). 
Por su parte, los nuevos partidos holandeses parecen haber surgido como respuesta a una amenaza que procede del exterior y que preocupa a los electores: la Unión Europea, el islam y la inmigración (Van Kessel, 2015). Solamente el FvD centra su discurso en la renovación democrática, al igual que Podemos y Cs en España. Mientras la crisis económica o sus efectos se mencionan en los programas españoles, en buena parte porque han sacado a relucir problemas de carácter estructural, en los programas holandeses apenas se nombran.

Si nos fijamos en las diferencias entre las dos grandes nuevas formaciones en España y en los Países Bajos, es decir, entre Podemos y el PVV, cabe concluir que su discurso apenas coincide a la hora de destacar cuáles son los asuntos prioritarios del país. Mientras el PVV se centra en los problemas causados por la islamización, la inmigración y el nacionalismo europeo (factores externos), Podemos se centra en la participación ciudadana, la igualdad, la solidaridad y la democratización (factores internos).

El surgimiento de nuevos partidos en los Países Bajos podría atribuirse a una respuesta ante los nuevos temas que generan conflicto político dividiendo a la sociedad y a los que los partidos tradicionales no habrían dado respuesta, como la integración europea, la inmigración o el choque con la cultura islámica. En España, Ciudadanos y Podemos habrían emergido para dar respuesta a los problemas del sistema político: las consecuencias de la falta de meritocracia (fraude y corrupción), los fallos en la participación democrática y la estructura administrativa y territorial del Estado (Rodríguez Teruel y Barrio, 2015; Rodón y Hierro, 2016). Es decir, los nuevos partidos han surgido ante los sentimientos de desconfianza de los ciudadanos hacia las formaciones establecidas.

Estos resultados estarían, a su vez, dándonos información acerca de en qué contextos es propicio que surjan nuevas fuerzas políticas. Si nos centramos en elementos institucionales, como han hecho gran parte de las investigaciones sobre nuevos partidos, parecería poco probable que un nuevo partido pudiese emerger en España - con un sistema electoral con una baja magnitud de distrito y una elevada desproporcionalidad electoral (Montero y Riera, 2009)—. Sin embargo, el proceso de distanciamiento de los votantes respecto a las fuerzas tradicionales habría propiciado el surgimiento de nuevas formaciones. En los Países Bajos, aun teniendo en cuenta las facilidades que da su sistema electoral para que una nueva formación pueda entrar en el Parlamento nacional, el análisis de contenido de los programas ha mostrado que detrás del nacimiento de nuevas formaciones estaría la inclusión de nuevos temas que suscitan interés entre parte del electorado no incorporados suficientemente en los programas políticos de los partidos establecidos.

Por lo general, estos hallazgos estarían subrayando la idea de que los nuevos partidos, tanto en España como en los Países Bajos, han aprovechado la ventana de entrada que había dejado la Gran Recesión para dar respuesta a cuestiones políticas que son anteriores a 2008: la crisis del sistema de partidos en el caso español, y una crisis cultural y de valores en el caso de los Países Bajos. En este sentido, nuestro estudio completaría aquellos trabajos 
(Harmel y Robertson, 1985; Mair, 1989; Rydgren, 2004) que, con una metodología cuantitativa, habrían señalado qué elementos de largo plazo relacionados con el sistema de partidos estarían detrás del nacimiento de nuevas formaciones.

\section{Referencias bibliográficas}

Alonso, S.; Volkens, A. y GÓMEZ, B. (2012). Análisis de contenido de textos politicos: Un enfoque cuantitativo (Cuadernos Metodológicos). Madrid: Centro de Investigaciones Sociológicas, 160.

ARZHeimer, K. (2009). «Contextual Factors and the Extreme Right Vote in Western Europe, 1980-2002». American Journal of Political Science, 53 (2), 259-275. $<$ https://doi.org/10.1111/j.1540-5907.2009.00369.x>

Bermeo, N. y Bartels, L. (2014). Mass Politics in Tough Times: Opinions, Votes and Protest in the Great Recession. Oxford: Oxford University Press. $<$ https://doi.org/10.1093/acprof:oso/9780199357505.001.0001>

Beyens, S.; Deschouwer, K.; Van Haute, E. y Verthe, T. (2015). «Born again, or born anew: Assessing the newness of the Belgian political party New-Flemish Alliance (N-VA)». Party Politics, 23 (4), 389-399. $<$ https://doi.org/10.1177/1354068815601347>

Bolin, N. (2007). «New Party Entrance: Analyzing the Impact of Political Institutions». Umea Working Papers in Political Science, 2.

BoomgaArden, H.G. y VliegentharT, R. (2007). «Explaining the Rise of Anti-Immigrant Parties: The Role of News Media Content in the Netherlands». Electoral Studies, 26 (2), 404-417. <https://doi.org/10.1016/j.electstud.2006.10.018>

BosCH, A. y DuRÁN, I.M. (2017). «How does economic crisis impel emerging parties on the road to elections?: The case of the Spanish Podemos and Ciudadanos». Party Politics, 1-11. <https://doi.org/10.1177/1354068817710223>

Casal BérTOA, F. y Weber, T. (2016). Restrained Change: Party Systems in Times of Economic Crisis. Comunicación presentada en EPOP 2016. $<$ https://doi.org/10.2139/ssrn.2826629>

DALTON, R. y WeLDON, S. (2006). «Public images of political parties: A necessary evil?». West European Politics, 28 (5), 931-951. <https://doi.org/10.1080/01402380500310527>

DassonNeVILLE, R. y HoOghe, M. (2017). «Economic indicators and electoral volatility: Economic effects on electoral volatility in Western Europe, 1950-2013». Comparative European Politics, 15 (6), 919-943. <https://doi.org/10.1057/cep.2015.3>

DE VRIES, C.E. (2007). «Sleeping Giant: Fact or Fairytale?: How European Integration Affects National Elections». European Union Politics, 8 (3), 363-385. <https://doi.org/10.1177/1465116507079546>

Emanuele, V. y Chiaramonte, A. (2016). «A growing impact of new parties: myth or reality?: Party system innovation in Western Europe after 1945». Party Politics. <https://doi.org/10.1177/1354068816678887>

GÓMEZ-Reino, M. y LlamaZAReS, I. (2015). New left populism in contemporary Spain?: The upsurge of Podemos. Trabajo presentado en la 22 International Conference of Europeanists, París, del 8 al 10 de julio. 
Harmel, R. (1985). "On the Study of New Parties». International Political Science Review, 6, 403-418. $<$ https://doi.org/10.1177/019251218500600403>

Harmel, R. y Robertson, J.H. (1985). «Formation and Success of New Parties». International Political Science Review, 6, 501-523. <https://doi.org/10.1177/019251218500600408>

Hauss, C. y Rayside, D. (1978). "The Development of New Parties in Western Democracies Since 1945». En: Maisel, L. y CoOper, J. (eds.). Political Parties: Development and Decay. Beverly Hills, CA: Sage.

HernándeZ, E. y KrIESI, Hanspeter (2016). «The Electoral Consequences of the Financial and Economic Crisis in Europe». European Journal of Political Research, 55 (2), 203-224. <https://doi.org/10.1111/1475-6765.12122>

Hino, A. (2006). New Parties' Success in Western Europe: A Comparative Analysis. Essex: University of Essex.

- (2012). New Challenger Parties in Western Europe: A Comparative Analysis. Londres: Routledge. <https://doi.org/10.4324/9780203130698>

Hooghe, L. y MARKS, G. (2017). «Cleavage theory meets Europe’s crises: Lipset, Rokkan, and the transnational cleavage». Journal of European Public Policy, 25 (1), 109-135. <https://doi.org/10.1080/13501763.2017.1310279>

Kriesi, H.; Grande, E.; Lachat, R.; Dolezal, M.; Bornschier, S. y Frey, T. (2008). West European Politics in the Age of Globalization. Cambridge: Cambridge University Press. <https://doi.org/10.1017/cbo9780511790720>

Klingemann, H.-D.; Hofferbert, R. y Budge, I. (1994). Parties, Policies and Democracy. Boulder: Westview Press.

KrippendorfF, K. (2004). Content analysis: An introduction to its methodology. Thousand Oaks, CA: Sage.

Lago, I. y Martínez, F. (2011). «Why new parties?». Party Politics, 17 (1), 3-20. <https://doi.org/10.1177/1354068809346077>

LAVEZzOlO, S. y RAmiro, L. (2017). «Stealth democracy and the support for new and challenger parties». European Political Science Review, 10 (2), 267-289. <https://doi.org/10.1017/S1755773917000108>

LeCompte, M. y SCHEnsul, J. (1999). Analyzing and interpreting ethnographic data. Oxford: Altamira Press.

LiNDVALL, J. (2014). «The electoral consequences of two great crises». European Journal of Political Research, 53 (4), 747-765. <https://doi.org/10.1111/1475-6765.12055>

LinZ, J.J. y Montero, J.R. (2000). "The Party Systems of Spain». En: KarVonen, L. y KuHnle, S. Old Cleavages and New Challenges Party Systems and Voter Alignments Revisited. Londres: Routledge, 150-196. <https://doi.org/10.4324/9780203469323_chapter_9>

LiPSET, S.M. y ROKKAN, S. (1967). Party Systems and voter alignments: Cross national perspectives. Nueva York: Free Press.

LuCArDie, P. (2000). «Prophets, Purifiers and Prolocutors: Towards a Theory on the Emergence of New Parties». Party Politics, 6 (2), 175-185. <https://doi.org/10.1177/1354068800006002003> 
Magalhaes, P. (2014). «Financial Crisis, Austerity, and Electoral Politics». Journal of Elections, Public Opinion and Parties, 24 (2), 125-133. <https://doi.org/10.1080/17457289.2014.887090>

Mair, P. (1989). "The Problem of Party System Change». Journal of Theoretical Politics, 1 (3), 251-276. <https://doi.org/10.1177/0951692889001003001>

- (1996). "Party Systems and Structures of Competition». En: LEDUC, L.; NiEMI, R.G. y Norris, P. (eds.). Comparing Democracies. Londres: Sage, 83-106.

- (1999). «New Political Parties in Established Party Systems: How Successful Are They?». En: Beukel, E.; Klausen, K.K. y Mouritzen, P.E. (eds.). Elites, Parties and Democracy. Odense: Odense University Press.

- (2002). «In the aggregate: Mass electoral behaviour in Western Europe, 1950-2000». En: Kerman, H. (ed.). Comparative Democratic Politics. Londres: Sage, 122-140.

- (2008). «Electoral Volatility and the Dutch Party System: A Comparative Perspective». Acta Politica, 43 (2-3), 235-253. $<$ https://doi.org/10.1057/ap.2008.1>

- (2015). Gobernando el vacio: La banalización de la democracia occidental. Madrid: Alianza Editorial. <https://doi.org/10.5209/poso.52946>

McMillan, J. y SCHumacher, S. (1993). Research in education: A conceptual understanding. Nueva York: Harper Collins.

Montero, J.R. y RierA, P. (2009). «El sistema electoral español: Cuestiones de desproporcionalidad y reforma». Anuario de la Facultad de Derecho de la Universidad Autónoma de Madrid, 13, 225-270. Recuperado de <http://afduam.es/wp-content/uploads/pdf/13/el-sistema-electoral-espanol-jose-ramon-montero.pdf>.

Morgan, J. (2013). Bankrupt Representation and Party System Collapse. University Park: Penn State University Press.

Orriols, L. y Cordero, G. (2016). «The Breakdown of the Spanish Two-Party System: The Upsurge of Podemos and Ciudadanos in the 2015 General Elections». South European Society and Politics, 21 (4), 469-492. <https://doi.org/10.1080/13608746.2016.1198454>

Pinard, M. (1975). Rise of a Third Party: A Study in Crisis Politics. Englewood Cliffs, NJ: Prentice Hall General.

RAmA, J. (2016). «Ciclos electorales y sistema de partidos en España, 1977-2016». Revista Jurídica: Universidad Autónoma de Madrid, 34, 241-266.

RoberTs, K.M. (2017). "Party politics in hard times: Comparative perspectives on the European and Latin American economic crises». European Journal of Political Research, 56 (2), 218-233. <https://doi.org/10.1111/1475-6765.12191>

Rodon, T. y Hierro, M.J. (2016). "Podemos and Ciudadanos Shake up the Spanish Party System: The 2015 Local and Regional Elections». South European Society and Politics, 21 (3), 339-357. <https://doi.org/10.1080/13608746.2016.1151127>

Rodríguez Teruel, J. y Barrio, A. (2015). «Going National: Ciudadanos from Catalonia to Spain». South European Society and Politics, 21 (4), 587-607. <https://doi.org/10.1080/13608746.2015.1119646>

Rodríguez-Teruel, J.; Barrio, A. y BarberÀ, O. (2016). «Fast and Furious: Podemos' Quest for Power in Multi-level Spain». South European Society and Politics, 21 (4), 561-585. <https://doi.org/10.1080/13608746.2016.1250397> 
ROURKe, L. y ANDERSON, T. (2004). «Validity in quantitative content analysis». Educational

Technology Research and Development, 52 (1), 5-18. $<$ https://doi.org/10.1007/bf02504769>

Rydgren, J. (2004). "Explaining the Emergence of Radical Right-wing Populism: The Case of Denmark». West European Politics, 27 (3), 474-502. <https://doi.org/10.1080/0140238042000228103>

Schumacher, G. y Rooduijn, M. (2013). «Sympathy for the "devil”?: Voting for populists in the 2006 and 2010 Dutch general elections». Electoral Studies, 32 (1), 124-133. <https://doi.org/10.1016/j.electstud.2012.11.003>

Segatti, P. y CAPUZZi, F. (2016). «Five Star Movement, Syriza and Podemos: A Mediterranean Model?». En: Martinelli, A. (ed.). Populism on the Rise, Democracies Under Challenge? Milán: Instituto per gli Studi di Politica Internazionale. Recuperado de <https://www.ispionline.it/sites/default/files/pubblicazioni/report_populism_2016_0_0.pdf>.

SIKK, A. (2005). «How unstable?: Volatility and the genuinely new parties in Eastern Europe». European Journal of Political Research, 44 (3), 391-412. <https://doi.org/10.1111/j.1475-6765.2005.00232.x>

Strauss, A. y CorbIn, J. (1998). Basics of Qualitative Research: Techniques and Procedures for Developing Grounded Theory. Londres: Sage Publications Ltd. <https://doi.org/10.4135/9781452230153>

TAvits, M. (2006). «Party System change: Testing a model of new party entry». Party Politics, 12 (1), 99-119.

<https://doi.org/10.1177/1354068806059346>

- (2007). «Party System in the Making: The Emergence and Success of New Parties in New Democracies». British Journal of Political Science, 38 (1), 113-133. <https://doi.org/10.1017/s0007123408000069>

Torreblanca, J.I. (2015). Asaltar los cielos: Podemos o la política después de la crisis. Barcelona: Debate.

VAN KesSel, S. (2015). «Dutch populism during the crisis». En: KrIESI, H. y PAPPAS, T.S. (eds.). Populism in the Shadow of the Great Recession. Colchester: ECPR Press, 109-124.

VIDAL, G. (2017). "Challenging business as usual?: The rise of new parties in Spain in times of crisis». West European Politics, 41 (2), 261-286. $<$ https://doi.org/10.1080/01402382.2017.1376272>

Vliegenthart, R.; BoOmgaarden, H.G. y Van Spanje, J. (2012). «Anti-immigrant Party Support and Media Visibility: A Cross-party, Over-time Perspective». Journal of Elections, Public Opinion and Parties, 22 (3), 315-358. $<$ https://doi.org/10.1080/17457289.2012.693933>

Volkens, Andrea; Lehmann, Pola; Matthiess, Theres; Merz, Nicolas; Regel, Sven y Wessels, Bernhard (2017). The Manifesto Data Collection: Manifesto Project Dataset (version 2017a). Berlín: Wissenschaftszentrum Berlin für Sozialforschung (WZB).

<https://doi.org/10.25522/manifesto.mpds.2017a> 
